\title{
SUBCUTANEOUS BASIDIOBOLOMYCOSIS: A CASE REPORT
}

\author{
Adziri Sackey $^{1}$, Nenyin Ghartey ${ }^{2}$ and Richard Gyasi ${ }^{3}$ \\ Ghana Med J 2017; 51(1): 43-46 DOI: http://dx.doi.org/10.4314/gmj.v51i1.9
}

\begin{abstract}
${ }^{1}$ Department of Child Health, School of Medicine and Dentistry, College of Health Sciences, P.O. Box 4236, Accra, Ghana, ${ }^{2}$ Children's Block, Korle Bu Teaching Hospital, P.O. Box 77, Korle Bu, Accra, ${ }^{3}$ Department of Pathology, School of Medicine and Dentistry, College of Health Sciences, PO Box GP 4236, Accra, Ghana
\end{abstract}

Corresponding author: Dr. Adziri Sackey

E-mail: sackey@sky.com

Conflict of interest: None declared

\section{SUMMARY}

Basidiobolomycosis is an uncommon chronic deep fungal infection in which gradually enlarging granulomas form, usually in the subcutaneous fat tissues of the limbs, chest or trunk of immunocompetent hosts, primarily children and young adults. It is caused by the fungus Basidiobolus ranarum. Definitive diagnosis is by microscopy and histopathology. Effective treatments include ketoconazole, itraconazole, potassium iodide and co-trimoxazole.

A 3 year old girl presented with ulcerations on the right thigh for one month, and painful swelling of the right leg and right buttock for six months. The right lower limb and vulva were swollen, tender and hard with hyperpigmentation and inguinal lymphadenopathy.

She had severe anaemia, eosinophilia and negative serology for HIV I and II. Histopathological examination showed a dermal chronic granulomatous infiltrate with fungal hyphae and yeast forms suggestive of Basidiobolus ranarum. There was marked reduction in right leg size and inguinal lymphadenopathy after several weeks of oral itraconazole, and complete healing of ulcers after 10 weeks.

The purpose of this report is to increase awareness of this disfiguring condition which is treatable but, if not correctly diagnosed, could result in inappropriate interventions such as amputation and anti-coagulant therapy.

Funding: None declared

Keywords: Deep fungal infection, basidiobolomycosis, itraconazole, chronic ulcer, leg swelling

\section{CASE REPORT}

A 3 year old girl from the Northern Ghana, West Africa, presented with a six month history of progressive painful swelling of the right leg and right buttock; and for one month, ulceration of the right thigh. She had had a recurrent small non-tender swelling on the right thigh that had been treated with a topical herbal preparation from the age of 1 year. There was a history of fever, chills, loss of appetite and difficulty walking. She lived in a rural area with her parents, both farmers, and three siblings. They had used leaves for cleaning after defecation.

On presentation, she was in pain, looked ill and pale but not jaundiced. Her growth was normal. There were distended superficial abdominal veins and hepatosplenomegaly.

Figure 1 shows the whole of the right lower limb was grossly swollen extending to the right vulva. The right leg measured $24.4 \mathrm{~cm}$ and the left leg $15 \mathrm{~cm}-$ measured from the tibial tuberosity. There were multiple ulcers on the right upper thigh, measuring between $2 \times 3 \mathrm{~cm}$ and 1 $x 1.5 \mathrm{~cm}$ with sloping edges, sloughy floor and serous discharge. The skin of the right leg was hyper pigmented with scarification marks. The right leg was warm, hard and tender to touch.

The right inguinal lymph nodes were matted and tender. There was reduced passive movement at the right ankle, knee and hip joints. The right dorsalis pedis was palpable but not the posterior tibialis. Peripheral oxygen saturation $(\mathrm{SpO} 2)$ was $98 \%$ in both lower limbs. Initial laboratory results showed a haemoglobin of $5.2 \mathrm{~g} / \mathrm{dl}$, total white cell count of $12.5 \times 10^{\wedge} 9 / \mathrm{L}$ with eosinophilia of $5.6 \%$ and normal platelet count. A blood film comment showed severe microcytic hypochromic anemia. There was no bacterial growth on blood culture. Xray of the right leg showed no bony abnormality. Doppler ultrasound of the right leg showed an echogenic thrombus in the proximal portions of the long saphenous, popliteal, common femoral, superficial and deep femoral veins. 


\section{Case Report}

The findings were reported to be consistent with deep vein thrombosis. Abdominal and pelvic ultrasound showed the liver to be diffusely echogenic and enlarged but no focal lesions were seen. The spleen measured $8.5 \mathrm{~cm}$ with a normal homogenous echo pattern and no focal lesions seen.

Swabs from the ulcers grew Citrobacter species sensitive to Levofloxacin. Serology was non-reactive for HIV I and II. Clotting screen was normal. Treatment was started with intravenous cloxacillin, crystalline penicillin, gentamycin and oral acetaminophen and haemotransfusions.

Despite local dressings with povidone-iodine and introduction of more antibiotics, metronidazole then clindamycin, the right leg and ulcers continued to increase in size. A differential diagnosis of deep fungal infection was considered, a biopsy was done and oral itraconazole $5 \mathrm{mg} / \mathrm{kg}$ started. A course of intravenous Amikacin was started following growth of Acinetobacter and Pseudomonas from a swab of the ulcers; local dressings were changed to potassium iodide solution, and supplementary vitamins and folic acid started.

The wedge biopsy was reported to have a creamy cut surface. On microscopy, the sections showed a dermal chronic granulomatous inflammatory infiltrate with fungal organisms. Figure 2 Grocott-Methenamine silver stain showed fungal hyphae and yeast forms. The hyphae were broad and septate with wide angle branching. The findings were consistent with Basidiobolus ranarum infection.

She was assessed by the plastic surgery service for consideration of skin grafting. The sizes of the ulcers now ranged between $10 \mathrm{~cm} \times 10 \mathrm{~cm}$ and $5 \mathrm{~cm} \times 5 \mathrm{~cm}$ but were clean and granulating. Wound dressings were changed to acetic acid and Nadoxin-impregnated Vaseline gauze.

Physiotherapy was arranged to help improve mobility particularly at the right ankle joint. She was discharged in the sixth week, and on review a week later, wound healing was progressing well. Skin grafting was delayed several times due to administrative issues, and by the seventh week of discharge the wounds had completely healed with minimal scarring. There was however an equinus deformity at the right ankle. The patient was subsequently lost to follow up.

Informed consent for clinical photographs was obtained from the child's mother and assent from the child. ${ }^{1}$

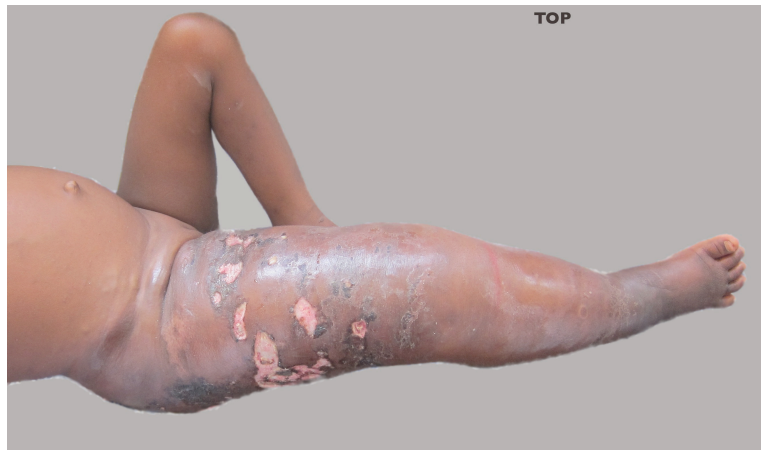

Figure 1 Grossly swollen right lower limb, inguinal area and buttocks with multiple ulcers and skin discolouration.

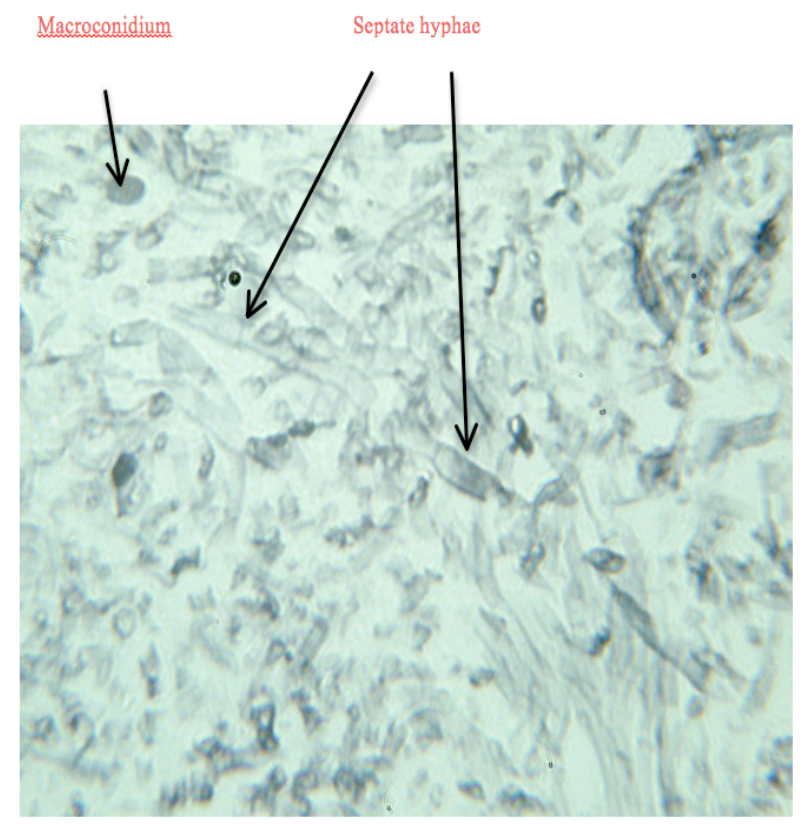

Figure 2 - Biopsy of subcutaneous tissue showing broad septate hyphae and macroconidia of basidiobolus ranarum. (Grocott-Methenamine silver stain, original magnification $\mathrm{x} 400)$.

\section{DISCUSSION}

Subcutaneous basidiobolomycosis is endemic to rural areas of the inter-tropical zone, particularly Indonesia, Burma, India, particularly South India, and sub-Saharan Africa. $^{2}$

The infection is caused by the filamentous fungus Basidiobolus ranarum, first described as an isolate from frogs in 1886 by Eidam. ${ }^{3} \mathrm{~B}$ ranarum is a fungus belonging to the class zygomycetes and order entomophthorales. Zygomycosis is an acute or chronic infection caused by fungal agents belonging to the phylum $\mathrm{Zy}-$ gomycota. 


\section{Case Report}

These are saprophytic fungi and are present in soil, decaying vegetable matter, and the intestines of amphibians, reptiles, fish and insectivorous bats. ${ }^{4}$

The precise mode of acquisition of $B$ ranarum is poorly understood but is thought to be acquired through skin after an insect bite, a scratch or minor cut. It may be picked up from decaying leaves used for cleansing after bowel movement, resulting in direct inoculation in the perineum (Mugerwa, 1976). ${ }^{5}$ Consistent with this theory is the fact that the thigh, buttock and perineum are frequent sites. Iatrogenic infection has also been reported. ${ }^{3}$

The first four human cases were reported in 1956 from Indonesia. ${ }^{6}$ In Africa, the condition was first reported in 1961, from Uganda where it appears to be widespread. ${ }^{7}$ Subsequently, reports were received from Sudan, Kenya, the Cameroons and Nigeria. Burkitt reports seeing a case in Accra, Ghana in 1964 in a 7 year old boy who had a typical lesion involving the buttocks and encircling the anal canal, producing a firm stricture. The diagnosis was confirmed on histology and culture of a surgical specimen.

Mugerwa reported 80 cases of subcutaneous phycomycosis infection in Uganda seen between 1967 and 1974. $76 \%$ of the patients were under 10 years of age, and $88 \%$ under 20 years of age. Mugerwa suggested that the use of 'toilet leaves' to clean after defecation might explain the observed distribution of lesions and source of infection.

No underlying disease appears to predispose to infection by $\mathrm{B}$ ranarum. ${ }^{8}$ Males are more frequently affected than females though this may be due to presentation bias. The perineum, buttocks, trunk and thighs are most frequently affected. Typically the presenting clinical feature is a firm subcutaneous painless indurated nodule on a lower extremity and buttock that gradually enlarges peripherally into a clearly defined flat mass attached to the overlying skin.

Sometimes the affected limb becomes swollen two or three fold. The overlying skin is intact with usually a bluish or purple colour (in light-skinned patients) at the growing region and is movable over the swellings. Pruritus or a burning sensation may develop in severe cases due to secondary infections. The swellings do not pit on pressure.

The joints are not directly affected but limb swelling may restrict movement. ${ }^{8}$ The infection is slowly progressive without treatment but may heal spontaneously. The nodule may ulcerate and cause lymphadenopathy but dissemination usually does not occur. When lym- phatics are involved, a non-pitting woody swelling results. Spontaneous resolution has been reported. Gastrointestinal, pulmonary, muscle or rhino-cerebral involvement is very rare. ${ }^{3}$

Anaparthy et al reported probably the youngest child with basidiobolomycosis. ${ }^{4}$ A six month old girl who presented with a four month history of slowly enlarging multiple painless swellings over her left knee preceded by an insect bite. She had multiple nodules over the extensor aspect of left thigh and a single well-defined erythematous indurated non-tender nodule of size $3 \mathrm{~cm}$ $\times 4 \mathrm{~cm}$ with central ulceration. The swelling was firm in consistency with smooth and rounded edges.

It was freely mobile over the underlying structure. Bilateral inguinal lymphadenopathy was noted. Following confirmation of basidiobolomycosis by culture and microscopy, the patient was put on saturated solution of oral potassium iodide (KI) in milk. The initial dose was 5 drops (approximately $1 \mathrm{ml}$ ) thrice a day after meals, increasing by 1 drop/dose until a dose of 40 drops/ dose was achieved. Therapy was continued until resolution of signs of active disease; dosage was then gradually discontinued. Complete resolution of the lesions occurred after eight weeks of treatment.

Mendiratta et al reported a case of a nine month old baby with rapidly progressing non-healing ulcers that spread to underlying muscles, mimicking a lymphoma. ${ }^{9}$ She responded poorly to itraconazole alone but showed dramatic improvement with a combination of itraconazole and potassium iodide. The case also highlights an early acquisition of the infection at one month of age.

Rodrigues and Oliver-Commey in 1994 reported four cases from Ghana; two boys and two girls ranging from three to six years of age. ${ }^{10}$ They all presented with extensive woody hard swellings which responded well to ketoconazole using a once daily treatment regimen.

Basidiobolomycosis may mimic other tropical infections that present with subcutaneous lesions. It may resemble other fungal infections (pythiosis and sporotrichosis), parasitic infections (filarial elephantiasis and onchocerciasis), bacterial infections (Mycobacterium tuberculosis and Mycobacterium ulcerans - buruli ulcer), and other diseases, including Burkitt's lymphoma and soft tissue tumour. ${ }^{11}$ Blood tests may show eosinophilia, high C-reactive protein (CRP) and erythrocyte sedimentation rate (ESR).

Definitive diagnosis is by microscopy and culture of clinical or surgical specimens. Histopathology demon- 
strates broad, thin-walled hyphae together with acute and/or chronic inflammatory cell infiltrates.

The organism does not typically invade blood vessels or other tissue; necrosis and tissue infarction are therefore not present.

Hyphae are easily visualized with hematoxylin and eosin stain. Microscopy shows beaked zygospores. Basidiobolus can be grown on standard mycology media such as Sabouraud, potato dextrose, and cornmeal agars. In addition to culture, infection with $\mathrm{B}$ ranarum may be diagnosed by an immunodiffusion test. ${ }^{12}$ This test is useful for monitoring infected patients and in making a diagnosis of disease even in the absence of culture.

Effective treatments include ketoconazole, itraconazole, fluconazole, potassium iodide and co-trimoxazole. A suggested dose of potassium iodide for children is $30 \mathrm{mg} / \mathrm{kg} /$ day for many months. With ketoconazole, itraconazole and fluconazole, liver enzymes must be monitored at least on a monthly basis. ${ }^{2}$ A reported regimen for co-trimoxazole is $80 \mathrm{mg}$ trimethoprim $+400 \mathrm{mg}$ suxamethoxazole per tablet -2 tablets a day in children for six to eight weeks. ${ }^{13}$

No single drug has proved effective in the treatment of all cases of basidiobolomycosis. Resistance to amphotericin has been reported in more than $50 \%$ of cases. Treatment should be continued for one month after lesions disappear. Patients treated with appropriate antifungals show complete resolution of the disease. Debridement is recommended by some for cutaneous disease but there is concern about surgery causing spread of the infection.

\section{CONCLUSION}

Basidiobolomycosis is an uncommon chronic deep fungal infection in which gradually enlarging granulomas form, usually in the subcutaneous fat tissues of the limbs, chest or trunk of immunocompetent hosts, primarily children and young adults. ${ }^{4}$ We present this report to increase awareness of a disfiguring condition which is treatable but, if not correctly diagnosed, could result in inappropriate interventions such as amputation and anti-coagulant therapy.

\section{REFERENCES}

1. Devakumar D, Brotherton H, Halbert J, Clarke A, Prost A, Hall J. Taking ethical photos of children for medical and research purposes in low-resource settings: an exploratory qualitative study. $B M C$ Med Ethics. 2013; 14: 27. Published online 2013 July 9. doi: 10.1186/1472-6939-14-27

2. Meunier YA. Deep fungal diseases. In: Tropical Diseases; A practical guide for medical practitioners and students. Oxford: Oxford University Press; 2013. p. 1-2.

3. Singh R, Xess I, Ramavat AS, Arora R. Basidiobolomycosis: A rare case report. Indian $\mathrm{J} \mathrm{Med} \mathrm{Mi}$ crobiol. 2008 Jul-Sep;26(3):265-7.

4. Anaparthy UR, Deepika G. A case of subcutaneous zygomycosis. Indian Dermatol Online J. 2014 Jan; 5(1):51 - 4 .

5. Mugerwa JW. Subcutaneous phycomycosis in Uganda. Br J Dermatol. 1976 May; 94(5):539-44.

6. Joe LK, Eng NIT, Pohan A, Van Der Muillen H, Emmons CW. Basidiobolus ranarum as a cause of subcutaneous mycosis in Indonesia. AMA Arch Dermatol. 1956 Oct;74(4):378-83.

7. Burkitt DP, Wilson AMM, Jelliffe DB. Subcutaneous phycomycosis: A review of 31 cases seen in Uganda. Br Med J. 1964 Jun;1(5399):1669-1672.

8. Gugnani HC. A review of zygomycosis due to Basidiobolus ranarum. Eur. J. Epidemiol. 1999 Nov; 15(10):923-929.

9. Mendiratta V, Karmakar S, Jain A and Jabeen M. Severe Cutaneous Zygomycosis due to Basidiobolus Ranarum in a Young Infant. Pediatric Dermatol. 2012 Jan-Feb;29(1):121-123.

10. Rodrigues O and Commey JOO. Basidiobolomycosis in Ghanaian children. Trop Doct. 1994 Oct;24:170-171.

11. Totten SP, Kumar V, Gupta A, Mallya A, Rao S. Subcutaneous phycomycosis - fungal infection mimicking a soft tissue tumor: a case report and review of literature. J Trop Pediatr. 2010 Feb; 56(1):65-6.

12. Nemenqani D, Yaqoob N, Khoja H, Al Saif O, Amra NK, Amr SS. Gastrointestinal basidiobolomycosis: An unusual fungal infection mimicking colon cancer. Arch Pathol Lab Med 2009 Dec; 133 (12):1938 - 42. Available from: http://www.archivesofpathology.org/doi/pdf/10.104 3/ $1543-2165-133.12 .1938$. [Last accessed 2013 Jan 31]

13. G.H. Hitchings, editor. Inhibition of Folate Metabolism in Chemotherapy: The Origins and Uses of Co-trimoxazole. Berlin: Springer Science \& Business Media, 2012. p. 41-42. 